\title{
"Due to the side effects she has when using the method it will be difficult for nurses to convince her to continue with that method. That's why I said she will leave with another method.": Family planning providers and contraceptive users in Rwanda do not consider discontinuation an option
}

Hilary M. Schwandt ( $\nabla$ Hilary.schwandt@wwu.edu )

Western Washington University https://orcid.org/0000-0003-3959-9594

Angel Boulware

Spelman College

Julia Corey

Wheaton College

Ana Herrera

Northwest Vista Community College

Ethan Hudler

Whatcom Community College

Claudette Imbabazi

INES

Ilia King

Xavier University

Jessica Linus

University of Maryland Baltimore County

Innocent Manzi

INES

Maddie Merritt

Western Washington University

Lyn Mezier

SUNY Oswego

Abigail Miller

Western Washington University

Haley Morris

Western Oregon University

Dieudonne Musemakweli 
INES

\section{Uwase Musekura}

Eastern Oregon University

\section{Divine Mutuyimana}

INES

\section{Chimene Ntakarutimana}

University of Kentucky

\section{Nirali Patel}

Arcadia University

\section{Adriana Scanteianu}

Rutgers The State University of New Jersey

\section{Biganette-Evidente Shemeza}

INES

\section{Madi Stapleton}

Western Washington University

\section{Gi'anna Sterling-Donaldson}

Drexel University

Chantal Umutoni

INES

Lyse Uwera

INES

\section{Madeleine Zeiler}

Western Washington University

Seth Feinberg

Western Washington University

\section{Research article}

Keywords: Rwanda, contraception, discontinuation, sub-Sahara Africa, switching

Posted Date: June 5th, 2019

DOl: https://doi.org/10.21203/rs.2.9944/v1

License: (c) (1) This work is licensed under a Creative Commons Attribution 4.0 International License. Read Full License 


\section{Abstract}

Background Contraceptive discontinuation is a common event. Family planning programs often focus more on recruitment of new users as opposed to maintaining use among current users. A focus on sustaining users is imperative for long-term family planning program success. Methods This qualitative study in Rwanda in 2018 included eight focus group discussions with family planning providers and 32 in-depth interviews with contraceptive users. The data were collected in the two districts with the highest and lowest rates of contraceptive use, Musanze and Nyamasheke, respectively. The aim of this study is to better understand contraceptive use dynamics in Rwanda. Results Family planning providers and current users in Rwanda do not consider method discontinuation an option. Providers give support and medicine for side effects for continuers and counseling for those opting to switch. Current users are willing to try many methods until they find the right one for them - and once they find the right method, they plan to, or use the method, for long durations. Conclusions The Rwandan family planning program is primed to meet the needs of users in their sustained use of contraceptives through empathetic and responsive counseling. Concurrently, family planning users are determined to use family planning to meet their desired family size goals. The matching goals of both providers and clients indicates that contraceptive use will likely continue to increase in the future in Rwanda; however, due to long periods of pregnancy prevention desired - increased use of long acting methods should be encouraged.

\section{Background}

Approximately 215 million women in the world wish to avoid or delay pregnancy yet do not use family planning methods (1). If family planning needs were fully met, there would be a $67 \%$ reduction in unintended pregnancies, $70 \%$ fewer maternal deaths, $44 \%$ fewer newborn deaths, and $73 \%$ fewer unsafe abortions (1).

Discontinuation of contraceptive methods, despite a desire to continue to avoid pregnancy, is very common, and is concerning for family planning programs. Researchers examining data from 36 developing countries estimated that approximately a third of unintended births are due to contraceptive discontinuation. Globally, that could mean that 25 million of the 75 million unintended births are due to contraceptive discontinuation (2). Studies report discontinuation rates at 6-56\% after just 12 months of contraceptive use (3-13). Discontinuation, while still in need of pregnancy protection, increases with time beyond the most common measure of after 12 months of use $(3,9,13)$. Discontinuation has been reported as significantly more common in sub-Saharan Africa as compared to other world regions (14). Often the most common reasons for discontinuation for those who wish to continue to prevent pregnancy is method related - such as health concerns or the experience of side effects $(3,7,9,12,15)$.

Switching from one method to another method is the ideal course of action for women and couples who desire to continue to prevent pregnancy but are not satisfied with their current method. In a study in Malawi switching within three months of discontinuation was just $4 \%$, while in a study in Kenya, $63 \%$ of discontinuers had switched to another method within three months of discontinuation (16)'(17). A study 
examining switching in 23 low and middle income countries found switching to be around $60 \%$ within three months (15). While researchers examining switching after one year of use found rates between $10 \%$ and $80 \% .(3,7,9,12)$ Researchers have noted that switching occurs at a lower level in sub-Saharan Africa countries than in other regions of the world (3).

Studying contraceptive use dynamics, such as discontinuation and switching, among contraceptive users becomes increasingly important in contexts where the desire for large family sizes are decreasing and contraceptive use is increasing. This profile fits the nation of Rwanda (18) - where mean ideal family size among all women declined from 4.3 to 3.3 between the years of 2005 and 2010. During the same period, modern contraceptive use among currently married women increased from $10 \%$ to $45 \%$. In 2015 , $28 \%$ of family planning users in Rwanda discontinued within 12 months of initiating use - with $34 \%$ discontinuing due to health concerns and side effects - the most common reason for discontinuation and $10 \%$ switched methods (19). As a result of these rapid changes in Rwanda and an interest in the role of contraceptive dynamics in the Rwandan family planning landscape, this study aims to understand the role of switching and discontinuation among family planning providers and experienced users in Rwanda.

\section{Methods}

This qualitative study took place in Rwanda in 2018 and included both focus group discussions and indepth interviews. Eight focus group discussions were held in February of 2018 with family planning providers and lasted on average for two hours. Four of the focus group discussions were conducted with family planning nurses and the other four were held with community health workers (CHW). The 32 indepth interviews were conducted in July of 2018 with mostly current modern contraceptive users who were at least 18 years old and lasted on average 43 minutes. Local NGO and government health staff helped recruit family planning nurses and CHWs. Subsequently, family planning nurse and CHW study participants helped to recruit contraceptive users.

The original study design included 16 current modern contraceptive users who had been using a modern method of contraception for at least six months and 16 women who had discontinued a modern contraceptive method within the last six months, for reasons other than desiring a pregnancy. During recruitment of contraceptive users it was apparent that the study definition of discontinuation was not clear - as women who had discontinued a hormonal method and then selected condoms were classified as discontinuers by the recruiters. Finding women who met the study definition of discontinuation was a challenge. By the end of the study, 13 "discontinuers" were included but of these 13 , six were currently using condoms.

Half of the focus group discussions and interviews took place in the Musanze district of Rwanda; the other half took place in Nyamasheke district. These two districts were selected purposively based on DHS data showing that Musanze was the district with the highest modern contraceptive prevalence rate and Nyamasheke was the district with the lowest (19). 
The focus group discussions were each led with a moderator who was assisted by a note-taker. The indepth interviews were conducted by one interviewer. All of the data collectors were fluent in Kinyarwanda

- the first language of all study participants. The data collectors completed an extensive training prior to data collection - that included review of study aims, research ethics, as well as extensive review and practice using the topic guides. Data collectors were trained to use the questions as a guide - not a survey, and to allow the conversations to flow. All data collection was done in Kinyarwanda. The data collection activities were recorded, with full consent of the study participants.

Ethical approval to conduct this study was obtained from the Institutional Review Boards at Western Washington University and the Ministry of Education of Rwanda. Participation in this study was completely voluntary. Every study participant read and signed a consent form prior to participation in the study. Study participants each received 10,000 Rwandan francs to cover transportation to and from the data collection venue.

Translation occurred during the transcription process from Kinyarwanda to English. The English transcripts were then loaded into Atlas.ti 8 (20) for coding. Teams of researchers each coded the same two transcripts - and then collaboratively created the universal codebook. The teams then used the codebook to code the remaining transcripts. Microsoft Excel spreadsheets were then created for each code to analyze the code for further subthemes, examine relationships within codes, and to analyze differences in themes by study demographics. Triangulation of data occurred between the data from the providers and the current users. Analysis was guided by the thematic content analysis approach (21).

\section{Results}

\section{Fears of Side Effects}

When discussing fears as related to contraceptive use, the main theme that arose among study participants was that of side effects. Fear of side effects was noted much more often in Nyamasheke than in Musanze. Fears about side effects surfaced for women prior to starting contraceptive use.

I have tried to fight against the feelings that using family planning will put me at risk.

Female, pills for 4 years, 30-35 years old, limiter

Some current users were still fearful that side effects could occur in the future.

I: Did you have any fears about the side effects of using this method?

R: Yes and I still have that fear. I think the fears will stop after the three years because I am still nervous about the side effects.

Female, implant for 6 months, 25-30 years old, spacer 
For others, the fear of side effects was realized and created fear about future experience of side effects, which impacted method choice.

I will not try another method because I am afraid of having other effects because the ones I tried didn't work. I will continue to use condoms.

Female, condoms for 10 years, 35-40 years old, limiter

Some participants noted how rumors spread about contraceptives induce fear for them, or others, in terms of family planning use.

I knew many rumors that said kids will grow funny if you use family planning and it will cause many side effects so I refused to use it.

Female, implant for 6 months, 25-30 years old, spacer

\section{Side Effect Experience}

Every study participant, provider and contraceptive user, contributed to the theme of side effects. Specific side effects mentioned, in order of frequency for contraceptive users, included: increased menstruation, dizziness, headache, amenorrhea, fatigue, weight gain, loss of libido, and weight loss. Nyamasheke contraceptive users made twice as many comments about side effects as compared to Musanze contraceptive users.

The experience I have gotten from the methods I was using was not good. From the first method (injectable for three months) I missed my period, I gained too much weight and I got back pain. Then from the implant I was bleeding - when I was having my period one week, after one week again I would have my period, so like 15 days in a month. The implant was the worst compared to the injectable. After that, the condoms I am using don't cause too much trouble with my health. I am getting my period regularly for three days or four and then it's gone. And also my weight has reduced.

Female, condoms for 10 years, 35-40 years old, limiter

Some women complained of side effects without being specific - while others noted serious side effects not typically associated with hormonal contraceptive use.

...when I used the injection I suffered from back pain and shivers in my body.

Female, former injectable user, 30-35 years, spacer

Study participants noted how people's bodies differ - so one person's difficulties with contraceptives may not necessarily be the experience of others. Women noted this as general advice - and also spoke about their personal experiences. 
...every woman responds differently to family planning methods.

Female, former implant user, 35-40 years, limiter

She has to talk to the doctor about which method she should use according to her unique body. There are women who use contraceptives who never have problems the whole time, and there are some women who have side effects after using contraceptives for a month.

Female, condom user for 2 years, 30-35 years old, limiter

I: How much longer do you plan on using this method?

R: I cannot say the exact time that I want to use this method because it is currently working well for my body and I think that I could continue using this method, unless there are any side effects that may come up, but other than that I will continue using it forever.

Female, injectable user for 6 years, 30-35 years old, spacer

There was an awareness that hormonal method misfit with a body would most likely occur at the beginning of method use; however, some women also noted how fit with the body can change after using a method for a long period of time.

By now, I can't predict the time for how much longer I will be using this method because now it has to do with my body, but maybe I may change after ten years when I see... when my body stops fitting to the method I am using.

Female, pills for 4 years, 30-35 years old, limiter

A common subtheme about side effects was the experience of not having any problems. Contributions to this theme of no side effects were nearly three times more common among Musanze participants than Nyamasheke participants.

The experience I had in using the injectable for 10 years is that I never had any side effects or any problem with that.

Female, implant user for 4 years, 30-35 years old, limiter

More commonly, the absence of side effects was noted as one aspect of their entire experience using contraceptives - but presented as though this was their main experience. Many women tried multiple methods before they found a method that worked for them - and sometimes a method that worked previously no longer worked after a break or after a period of sustained use.

After three years of using the injectable, I started seeing changes, like during my menstruation period I started to get dizzy and fall down. 
Female, condoms, 35-40 years old, limiter

Sometimes women would report experiencing difficult side effects, and having no side effects, in the same interview. At times even when reporting about experiences using the same method - as demonstrated by this participant about her experiences with injectables early in the interview:

I was bleeding so much and I lost weight and I was about to faint.

Then later in the interview, she has this to say:

Until now, I haven't had any problems or side effects, and I don't think that I will have any problems in the future. Every time I go to see the doctor to check if everything is okay, it is.

Female, injectables for 10 years, $45-50$ years old, limiter

Providers and contraceptive users noted that women should return to the clinic when they experience unmanageable side effects as opposed to turning to others in the community for support, which most often leads to rumors. The advice to return to the clinic was made more often by nurses than $\mathrm{CHWs}$.

The advice I would give her is that she should not ignore it. I would tell her that if she has those side effects she should go to see a nurse who will try to find a way to help her.

Female, condoms, 35-40 years old, limiter

If your body is not responding well, you have to go to the health center to get help because if she doesn't go to a doctor, she goes to the neighbors or other women, is where she will get rumors that the contraceptive method is not good.

Nurse, female, 45-50 years old, sterilized

\section{Providers Help Prep Clients for Side Effects}

Providers and contraceptive users both spoke about the need to warn clients about potential side effects prior to use.

Before I started using family planning, they told us about all methods and they told us about the impact we can have from those methods. The injectable was the choice I made. After, I had side effects from the injectable but I knew it would happen because they told us about it.

Female, condoms for 5 years, 35-40 years old, limiter

R: From the day I started the injectable I didn't get my period.

I: Did you have a problem not having your period? 
R: I had no problem with that because the nurses and doctors told us to expect that.

Female, condoms for 5 years, 35-40 years old, limiter

Family planning users often shared how family planning providers also assisted new adopters during initial counseling to prepare for switching if side effects became unbearable.

...the community health workers told us when one method becomes too difficult to continue using you can shift methods.

Female, sterilized, 40-45 years old, limiter

Family planning providers warned new users about the potential for side effects and switching - they also helped facilitate continued use among users via reminders about upcoming appointments.

Contraceptive users described how the family planning providers would give them appointment times to return for resupply of their contraceptive method. Some women discussed the efforts that providers, particularly CHWs, would go to if women missed their appointments. They would remind the woman to come for the appointment - most often with a phone call.

...when that appointment comes, they (providers) reach us so we cannot miss our method.

Female, condom user, 35-40 years old, spacer

There was a tremendous amount of effort on the part of CHWs to make sure women knew when to follow up with appointments to avoid issues of unplanned pregnancies or the need for testing for pregnancy if too much time elapsed between the use. The focus was entirely on pregnancy - not on mitigation of side effects.

They gave use the date to come to take the injectable, so we try not to miss that date in order to avoid unplanned pregnancy. And ourselves, we try to respect that day because when you miss that day, you get many challenges in your life.

Female, injectable for 7 years, 30-35 years old, spacer

\section{Switching Hormonal Methods}

The topic of switching methods of contraception arose often with study participants - significantly more often than the topic of discontinuation. Providers and women describe the most common switching scenario as a desire to stop using their method of contraception due to unmanageable side effects.

First, I was using the three-month injectable, and I only used it for three months and then I had side effects. I found that this method didn't work with me well and I switched to pills, and I am using pills now. 
Female, pill user for 3 years, $40-45$ years old, spacer

Providers often responded to this issue with a desire to educate the client on their other options and to assist them in identifying a new method. Providers rarely mentioned the option for women to discontinue.

Due to the side effects she has when using the method it will be difficult for nurses to

convince her to continue with that method. That's why I said she will leave with

another method.

CHW, male, 50-55 years old, non-user

I tried to talk to a doctor, and asked them, "Since injections gave me lots of problems, what else can you offer?"...The nurses gave me advice to switch methods and go to the pills, and they said that if the pills didn't work for me that I can come back and see what else I can use.

Female, pill user for 10 years, 35-40 years old, limiter

Less often providers noted how they would work with the client to continue use of their current method, always noting how switching methods was an alternative if their efforts to mitigate side effects failed. Again, discontinuation was not offered as option in these scenarios.

Just because she experiences a side effect does not mean we must change the method of which we have used. As providers we have ways to treat various side effects, so that she may continue to use that method. However, if it is not treatable we will consider other contraceptive methods.

Nurse, female, 35-40 years old, implant user

For some current users interviewed, the amount of methods the women had tried, and switched to, was high. In our sample, two women had tried four methods. The average number of methods used for the entire sample was two. In contrast, some women were reluctant to switch methods if they were not experiencing side effects. In fact, half of our sample used only one method for, on average, seven years most often injectables. Sustaining use with the same method for long durations of time was more common in Musanze than in Nyamasheke.

I don't want to switch because I have never had side effects so I do not want to take the risk, maybe if I switch, the other method I will try will be bad.

Female, injectable user for 10 years, 35-40 years old, spacer

I will always use this method of pills, maybe forever ...I don't have plans to change or to switch to another method because these pills work well for me. If these pills work for me why would I change to another method? I will stay with this one. 
Female, pill user for 3 months, 30-35 years old, spacer

Providers and women also noted that women sometimes switched from shorter term methods to longer term methods - less so for increased effectiveness but more for the duration of contraceptive coverage particularly to limit the need to visit a health provider at regular intervals to increase economic opportunities.

The reason why she might want to stop the pill is because all the time she was going back and forth to the hospital, she could be doing something for her family. Instead of being in the same situation, she chose to use a longer method.

CHW, female, 45-50 years old, non-user

Providers were eager to learn from clients about their plans to discontinue and their reasons so they could properly counsel them on all of their options. Providers sought to assist women in continuing to use contraception when the women desired pregnancy prevention. It takes time for family planning providers to counsel dissatisfied users - to help them find solutions with their current methods or to educate clients about alternative methods. Providers did not explicitly state this time burden, nor identified it as a burden, but through their comments on the subject it is clear the efforts to help dissatisfied users can take significant time.

After understanding how all the different contraception methods can be used, she can then find one more suitable if she chooses to quit the first one.

CHW, female, 50-55 years old, injectable user

If I understand why she stopped using the contraceptive method I can start over by teaching her again about contraceptives and see if I can change her mind from the decision she made to stop using the contraceptive method.

Nurse, female, non-user

Family planning providers discussed these counseling sessions with empathy and understanding - and showed regret that they did not have more tools at their disposal to ease the burden of the side effects on their clients.

As she goes to the CHW to change the method, the CHW will not tell her this is the best method for you. They will explain to her again about other methods and she will choose a method on her own that she wants to use.

Nurse, female, implant user

What the CHW should do is give her time to explain and pay attention to what she is saying. Maybe she should come when there are less clients based on the appointment she is given so that the CHW has 
enough time to meet with her. It will allow her enough time to feel free to express those challenges and be given another method.

CHW, female, 45-50 years old, condom user

\section{Temporary Break from Hormonal Methods}

Side effects was the main reason study participants noted recent, or past, discontinuation of contraceptive methods. The discontinuation of contraception was always noted as a temporary break when due to unmanageable side effects.

I stopped using family planning in order to give my body a break, and I plan to continue using family planning after that break...

Female, traditional methods, 30-35 years old, spacer

Contraceptive users stated they discontinued - but actually they often switched from a hormonal method to a non-hormonal method - either condoms or traditional methods. Study participants viewed only hormonal methods as contraceptive methods.

I stopped using them because I had headaches and there were times when I felt like I couldn't see clearly. For now, I am using condoms, but because I had those headache and vision problems I stopped the implant. I stopped because I had those side effects, but when the problems subside I will use another type of contraception.

Female, condom user for 2 years, 30-35 years old, limiter

Study participants noted through interactions with providers that the providers tried to help alleviate the side effects, and when that did not work, advised the users to pick a method with no side effects in the interim period between discontinuing their hormonal method and selecting a different hormonal method.

l: Why did you discontinue this method of using pills?

R: This method of pills caused me to have more stress, headaches, amnesia, and bleeding. Then I decided to discontinue, and I went to the health center and after testing me they told me that I had to use condoms or the calendar method while I thought about another method I could use.

Female, condom user for 6 years, 35-40 years old, limiter

Before I discontinued, I went to the health center and they gave me some drugs because I was having more bleeding and high blood pressure with the five-year implant, but there was no change. ... And then, the nurses and doctors helped me take out the implant. They advised me to go and take some rest and to use condoms and the calendar method while I thought about the method that I may use next. 
Female, former implant user, 35-40 years, limiter

Actual discontinuation was most often related to unmanageable side effects.

Maybe this woman has gotten bad side effects from every method she has tried, so there is not another option.

CHW, male, 40-45 years old, non-user

\section{Persistence Advice}

Study participants noted how they often advise their friends and neighbors to stick with family planning use despite the issues that arise with hormonal contraceptive use - that the side effects would pass with time so they should persist in using the method of family planning they were currently using.

...I tell them don't be discouraged, continue using family planning.

Female, injectable for 25 years, 45-50 years old, limiter

If you find that none of those methods work for you, you can ask a nurse for advice, but you cannot start using contraceptives for two months and then say that you are going to stop.

Female, condoms, 35-40 years old, limiter

Women wanted to encourage others to recognize that bodies differ - and promote use for others through an awareness of finding the right method for your body type, without giving up during this process of searching.

For me, when I used injections I did not have good luck with them, but I would say to them that they might be good for you, and if using injections doesn't work for you, then you can use pills.

Female, pill user for 10 years, 35-40 years old, limiter

Another piece of advice for someone who has side effects is that she doesn't have to stop completely in fear that if she uses another method it will cause problems. I think she should try and try until she gets the type of contraceptive that fits her body.

Female, pill user for 3 years, 40-45 years old, spacer

\section{Persistence}

The topic of discontinuation arose infrequently. Providers spoke about when women choose to use contraception to either limit or space their births, then the only response to this desire was to find a 
method that worked to assist the client in achieving this goal.

If someone wants to stop using family planning, if they come to me as a CHW I will show them first the world they live in and how small it actually is. And then I will show her how difficult it is to find her child milk.... I will let her make the decision of using family planning or not. In short, I will say teaching her so that she might not be disappointed in stopping and I will give her examples by showing her her neighbors and friends and after that she might have courage in family planning.

CHW, male, 55-60 years old, implant user

A few providers noted how they would warn clients about the potential consequences of discontinuation when still desiring to avoid and unwanted pregnancy.

I would advise them about the side effects they can face if they stop using family planning. When you don't use family planning you can have too many kids to care for based on your financial circumstance, they may face malnutrition.

CHW, female, 40-45 years old, injectable user

Well over half of respondents expressed a desire to continue using family planning despite facing side effects related to their contraceptive use. Respondents who reported this persistence were often pushing through side effects caused by their contraceptive method; however, despite these side effects, the respondents were adamant about continuing to use family planning.

I went to him (CHW) and tried to discuss my problems with him, and I tried to show him the difficulties I have with using pills... but also that I didn't want to stop using them.

Female, pill user for 10 years, 35-40 years old, limiter

I was bleeding so much and I lost weight and I was about to faint. The doctor told me to go home and continue using the pull-out method. That is when I refused to go home and I insisted that they give me the injectable again.

Female, injectables for 10 years, $45-50$ years old, limiter

Respondents noted how finding ways to deal with the side effects of contraceptives was much better than facing the alternative of not using family planning.

... even though I had those side effects when I started using family planning, if I had given up using family planning in the first place I would have more children so I continued to use it even though I knew that I was passing through a hard time with the side effects until we had the children we wanted to have.

Female, condoms for 10 years, 35-40 years old, limiter 
...using injectables helped me to not get pregnant when I didn't want to get pregnant. I was met with side effects but the injectable helped me to not get pregnant.

Female, pill user for 3 years, $40-45$ years old, spacer

\section{Conclusion}

In this study, discontinuation was noticeable only in its absence as an option for dissatisfied contraceptive users by both family planning providers and experienced contraceptive users. Once a couple decides to use contraception to achieve their spacing or limiting goals, their providers and support systems aim to help them achieve those goals through supporting sustained use or switching methods, despite the experience of challenging side effects. Similar themes were noted in one study in Uganda (22).

As with contraceptive users worldwide, participants in this study were most fearful of side effects when contemplating use of contraceptives - and even during use (4,22-25). The actual experience of side effects with contraceptive use was incredibly common, and rare was the experience of no side effects with contraceptive use; however, some with negative experiences of side effects expressed a desire to downplay the negativity and present their experience as overall positive.

Contraceptive users and family planning providers alike noted how helpful providers can be in warning new users about the likely experience of side effects - as well as with switching if side effects became unbearable. Many researchers have concluded the importance of quality family planning services - in particular, family planning provider counseling on the anticipated side effects and switching as one deterrent to contraceptive discontinuation $(2-4,6,7,10,11,14,22-28)$; while others suggest that programs and providers have little impact on continuation $(13,17)$. One study analyzed the correlation between discontinuation rates and family planning program effort scores, and found a negative relationship, albeit weak, and concluded that family planning programs can impact discontinuation, particularly in terms of training and supervision of providers (5). Another study tested a client-centered approach to family planning counseling and found that the intervention was successful in increasing provider, as well as client, knowledge of methods and side effects (29). Others found lower discontinuation rates among clients who felt they were adequately counseled as compared to other clients who felt they were not adequately counseled $(26,27,30,31)$.

Switching of contraceptive methods was common among some of the study participants. Participants also describe taking "temporary breaks" from hormonal methods. These breaks were described as a break from using family planning methods, even though they often entailed switching to less effective methods. A similar finding emerged from research in Ghana (24). Along these lines, the study design was not executed as planned due to different definitions of modern methods and discontinuation by the researchers and the study participant recruitment partners. 
Both contraceptive users and providers alike cautioned against women experiencing side effects turning to their friends and community networks for assistance - instead the overwhelming advice was to return to the professionals for help. Researchers have shown the impact of myths on contraceptive use $(4,22,23,32,33)$. Myths about contraceptive use were nearly absent from both the focus group discussions and the in-depth interviews - the emphasis lied more in avoiding myths and rumors than in actual discussion of what the myths were. By doing so, the study participants believed that women would be less likely to be negatively affected by myths and rumors about contraceptives - and by extension, would remain more successful in continuing to use contraception. Research in the Democratic Republic of Congo has demonstrated the positive impact of clients returning to providers for assistance with side effects instead of community members (7).

To avoid side effects, some study participants were reluctant to change methods. Given the long durations of contraception coverage desired by Rwandan women, who marry relatively young and have smaller family size desires, the need for long periods of protection should be matched by the use of longer acting methods. The Rwandan Family Planning program could improve, and serve women better, if women who need longer periods of protection were encouraged to use longer acting, and even permanent, methods.

When examining the differences in results by study district - the results align with what one would expect. In Nyamasheke, a district with lower prevalence of contraceptive use - the comments about side effect experiences were much more frequent. In contrast, in Musanze, where the contraceptive prevalence is higher - family planning users seem to be more comfortable with the issue of side effects, more likely to identify as not having side effects - even if they did note experiences with side effects in a different part of the interview, and more likely to be interested in switching for reasons other than experience of side effects, such as switching from short to longer acting methods.

A strength of the study is the inclusion of the perspectives of both family planning providers, at both the health center and community levels, as well as current contraceptive users. Study participants were drawn from two different districts in Rwanda - purposively selected for having the lowest and highest modern contraceptive prevalence in the nation. Finally, this study provides a qualitative addition to the predominately quantitative literature on discontinuation.

One limitation of the qualitative data is that results are not generalizable to the nation. In addition, former dissatisfied users were not included in the study at the level desired. Finally, current family planning health providers were used to recruit current contraceptive using study participants - so they likely selected clients they had positive and close relationships with.

Both contraceptive users and family planning providers present contraceptive use in Rwanda as an exercise in persistence. Once women have made the choice to use contraception to assist in planning their families - most are committed to finding a way to use contraceptives to achieve their goal. Family planning providers are eager to assist women in finding the means to do so as well - and seem to take on this role eagerly without complaint about the time and energy it takes to assist contraceptive users 
persist with contraceptive use through unmanageable side effects. Study participants showed, albeit not always explicitly, an awareness that the experience of negative side effects with contraceptives paled in comparison to the negative experience of having children when children were not desired. Given the willingness to persist, and not view discontinuation as an option, sustaining contraceptive use in Rwanda looks very promising as the support systems and structures are in place to assist women in continuing with contraceptive use once they begin.

\section{Abbreviations}

$\mathrm{CHW}=$ community health worker

\section{Declarations}

\section{Ethics approval and consent to participate}

Ethical approval to conduct this study was obtained from institutional review boards at Western Washington University and at the Rwandan Ministry of Education. Every subject participant read and signed a written consent form prior to participation.

\section{Consent for publication}

Not applicable.

\section{Availability of data and material}

The transcripts analyzed for the current study are available from the corresponding author on reasonable request.

\section{Competing interests}

The authors declare that they have no competing interests.

\section{Funding}

This study was funded by the National Science Foundation. The National Science Foundation had no role in the design of the study, collection of the data, analysis, interpretation of the data, nor writing the manuscript.

\section{Author's contributions}


HMS designed the study. AB, JC, AH, EH, CI, IK, JL, IM, MM, LM, AM, HM, DM, UM, DM, CN, NP, AS, BS, MS, GS, CU, LU, MZ, SF, and HMS analyzed the data. All authors read and approved the final manuscript.

\section{Acknowledgements}

The authors wish to acknowledge Dean Faustin Habineza for his role in enabling this international research collaboration as well as the individuals who helped recruit study participants for this study.

\section{References}

1. Singh S, Darroch JE, Ashford LS, Vlassoff M. Adding it up: The costs and benefits of investing in family planning and maternal and newborn health. New York: Guttmacher Institute and United Nations Population Fund; 2009.

2. Jain AK, Winfrey W. Contribution of Contraceptive Discontinuation to Unintended Births in 36 Developing Countries: Contribution of Contraceptive Discontinuation to Unintended Births. Studies in Family Planning. 2017 Sep;48(3):269-78.

3. Ali MM, Cleland J, Shah IH. Causes and consequences of contraceptive discontinuation: evidence from 60 Demographic and Health Surveys. Geneva, Switzerland: World Health Organization; 2012.

4. Alvergne A, Stevens R, Gurmu E. Side effects and the need for secrecy: characterising discontinuation of modern contraception and its causes in Ethiopia using mixed methods. Contraception and Reproductive Medicine [Internet]. 2017 Dec [cited 2018 Nov 3];2(1). Available from:

http://contraceptionmedicine.biomedcentral.com/articles/10.1186/s40834-017-0052-7

5. Blanc AK, Curtis SL, Croft TN. Monitoring Contraceptive Continuation: Links to Fertility Outcomes and Quality of Care. Studies in Family Planning. 2002;33(2):127-40.

6. Bradley SEK, Schwandt HM, Khan S. Levels, Trends, and Reasons for Contraceptive Discontinuation. Calverton, Maryland, USA: ICF Macro; 2009. (DHS Analytical Studies). Report No.: 20.

7. Casey SE, Cannon A, Mushagalusa Balikubirhi B, Muyisa J-B, Amsalu R, Tsolka M. Twelve-month contraceptive continuation among women initiating short- and long-acting reversible contraceptives in North Kivu, Democratic Republic of the Congo. Novick G, editor. PLOS ONE. 2017 Sep 8;12(9):e0182744.

8. Curtis S, Evens E, Sambisa W. Contraceptive Discontinuation and Unintended Pregnancy: An Imperfect Relationship. International Perspectives on Sexual and Reproductive Health. 2011 Jun;37(02):058-66.

9. Curtis SL, Blanc AK. Determinants of Contraceptive Failure, Switching, and Discontinuation: An Analysis of DHS Contraceptive Histories. Calverton, Maryland, USA: Macro International Inc.; 1997. Report No.: No.6. 
10. Modey EJ, Aryeetey R, Adanu R. Contraceptive Discontinuation and Switching Among Ghanaian Women: Evidence from the Ghana Demographic and Health Survey, 2008. African Journal of Reproductive Health. 2014;18(1):84-92.

11. Mumah JN, Machiyama K, Mutua M, Kabiru CW, Cleland J. Contraceptive Adoption, Discontinuation, and Switching among Postpartum Women in Nairobi's Urban Slums. Studies in Family Planning. 2015 Dec;46(4):369-86.

12. Steele F, Curtis SL, Choe M. The Impact of Family Planning Service Provision on Contraceptive-use Dynamics in Morocco. Studies in Family Planning. 1999;30(1):28-42.

13. Tolley E, Loza S, Kafafi L, Cummings S. The Impact of Menstrual Side Effects on Contraceptive Discontinuation: Findings from a Longitudinal Study in Cairo, Egypt. International Family Planning Perspectives. 2005 Mar;31(01):15-23.

14. Jain AK, Obare F, RamaRao S, Askew I. Reducing Unmet Need by Supporting Women with Met Need. International Perspectives on Sexual and Reproductive Health. 2013 Sep;39(03):133-41.

15. Ali MM, Cleland J. Contraceptive Switching after Method-related Discontinuation: Levels and Differentials. Studies in Family Planning. 2010 Jun 7;41(2):129-33.

16. Mumah JN, Machiyama K, Mutua M, Kabiru CW, Cleland J. Contraceptive Adoption, Discontinuation, and Switching among Postpartum Women in Nairobi's Urban Slums. Studies in Family Planning. 2015 Dec;46(4):369-86.

17. Dasgupta AN, Ngwalo R, Branson K, Gondwe L, Taulo F, Ngwira B, et al. Using patient-held records to evaluate contraceptive use in Malawi. Bulletin of the World Health Organization. 2015 Nov 1;93(11):76874.

18. Schwandt HM, Feinberg S, Akotiah A, Douville TY, Gardner EV, Imbabazi C, et al. "Family planning in Rwanda is not seen as population control, but rather as a way to empower the people": examining Rwanda's success in family planning from the perspective of public and private stakeholders. Contraception and Reproductive Medicine [Internet]. 2018 Dec [cited 2019 Jan 29];3(1). Available from: https://contraceptionmedicine.biomedcentral.com/articles/10.1186/s40834-018-0072-y

19. National Institute of Statistics of Rwanda, Ministry of Finance and Economic Planning, Ministry of Health, The DHS Program, ICF International. Rwanda Demographic and Health Survey, 2014-15: Final Report. Kigali, Rwanda: Rockville, Maryland, USA; 2016. 615 p.

20. Atlas.ti. Berlin: Scientific Software Development; 1993.

21. Green J, Thorogood N. Qualitative Methods for Health Research. Thousand Oaks: Sage; 2004. 
22. Kibira SPS, Muhumuza C, Bukenya JN, Atuyambe LM. "I Spent a Full Month Bleeding, I Thought I Was Going to Die..." A Qualitative Study of Experiences of Women Using Modern Contraception in Wakiso District, Uganda. Mintzes B, editor. PLOS ONE. 2015 Nov 2;10(11):e0141998.

23. Diamond-Smith N, Campbell M, Madan S. Misinformation and fear of side-effects of family planning. Culture, Health \& Sexuality. 2012 Apr;14(4):421-33.

24. Hindin MJ, McGough LJ, Adanu RM. Misperceptions, misinformation and myths about modern contraceptive use in Ghana: Table 1. Journal of Family Planning and Reproductive Health Care. 2014 Jan;40(1):30-5.

25. Sedgh G, Hussain R. Reasons for Contraceptive Nonuse among Women Having Unmet Need for Contraception in Developing Countries. Studies in Family Planning. 2014 Jun;45(2):151-69.

26. Cotten N, Stanback J, Maidouka H, Taylor-Thomas JT, Turk T. Early Discontinuation of Contraceptive Use In Niger and The Gambia. International Family Planning Perspectives. 1992 Dec;18(4):145.

27. Koenig MA, Hossain MB, Whittaker M. The Influence of Quality of Care upon Contraceptive Use in Rural Bangladesh. Studies in Family Planning. 1997 Dec;28(4):278.

28. Odwe G, Mumah J, Obare F, Wamukoya M, Machiyama K, Cleland J, et al. Factors influencing satisfaction with oral contraceptive pills and injectables among past users in Kenya. Journal of Biosocial Science. 2018 Oct 12;1-14.

29. Costello M, Lacuesta M, RamaRao S, Jain A. A Client-centered Approach to Family Planning: The Davao Project. Studies in Family Planning. 2001 Dec;32(4):302-14.

30. RamaRao S, Lacuesta M, Costello M, Pangolibay B, Jones H. The Link between Quality of Care and Contraceptive Use. International Family Planning Perspectives. 2003 Jun;29(2):76.

31. Sanogo D, RamaRao S, Jones H, N'diaye P, M'bow B, Diop CB, et al. Improving Quality of Care and Use of Contraceptives in Senegal. African Journal of Reproductive Health. 2003 Aug;7(2):57.

32. Ankomah A, Oladosu, Anyanti. Myths, misinformation, and communication about family planning and contraceptive use in Nigeria. Open Access Journal of Contraception. 2011 Jun;95.

33. Gueye A, Speizer IS, Corroon M, Okigbo CC. Belief in Family Planning Myths at the Individual and Community Levels and Modern Contraceptive Use in Urban Africa. International Perspectives on Sexual and Reproductive Health. 2015 Dec;41(04):191-9. 